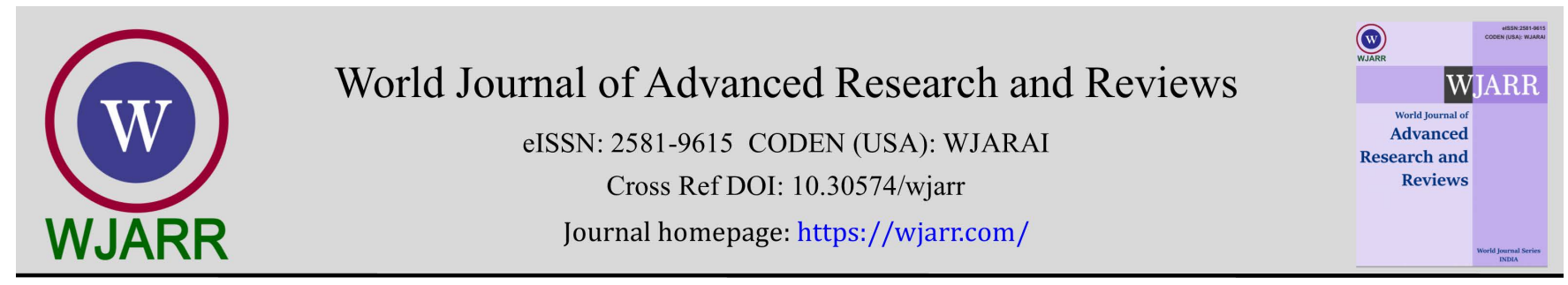

(REVIEW ARTICLE)

Check for updates

\title{
Life of patients with cerebellar ataxia: A systematic review
}

Débora Maria Figueiredo Lucena 1,*, Jéssika Figueiredo Lucena 2, Ana Kitéria Pinheiro Cavalcante 1, Alessandra Jespersen de Athayde Rocha ${ }^{1}$, Beatriz Nunes Ferraz de Abreu Zech Sylvestre ${ }^{1}$ and Laís de Miranda Sales Rocha ${ }^{1}$

${ }_{1}$ Medical student at the University Center Christus, Fortaleza, Ceará, Brazil.

${ }^{2}$ Medical student at the University Center Facisa, Campina Grande, Paraíba, Brazil.

World Journal of Advanced Research and Reviews, 2021, 12(03), 271-276

Publication history: Received on 08 November 2021; revised on 13 December 2021; accepted on 15 December 2021

Article DOI: https://doi.org/10.30574/wjarr.2021.12.3.0690

\begin{abstract}
Objectives: Conduct a literature search for scientific tests concerning treatment combined with well-being in cerebellar ataxias using electronic data sources.

Methods: A search was conducted organized by scientific tests worried about the treatment allied to well-being in cerebellar ataxias using the electronic data sources PubMed, Medline, Embase, Cinahl and Pedro, and listings of recommendations of articles, from 1980 to December 2011, including in English and Dutch.
\end{abstract}

Results: Information suggests that physical treatment, when included in occupational treatment, may improve international practical reputation, just as treatment at work alone may decrease signs of anxiety (grade 3).

Conclusion: We have found some care for performing physical and work treatment, but other studies are necessary to create medical technique suggestions.

Keywords: Ataxia; Cerebellar ataxia; Allied health; Physical treatment; Organized evaluation

\section{Introduction}

Ataxia is a neurological sign and symptom identified by loss of control of activities. The term cerebellar ataxia is used to suggest ataxia which is due to brain disorders, which leads to disturbances in the gait, balance and control, dominance, eye movements and speech [1-3].

There are numerous reasons for cerebellar ataxia, but one picture is degenerative cerebellar ataxias. For many people with ataxia, no matter the reason, there are no drug therapy alternatives. Physical treatment, speech treatment and work-related treatment are expected to eliminate second problems and reduce dependence on the everyday life of clients with ataxia.

As an initial step toward a much more evidence-based technique, we methodically evaluated the evidence for allied health treatments in cerebellar ataxia.

\footnotetext{
${ }^{*}$ Corresponding author: Débora Maria Figueiredo Lucena

Medical student at the University Center Christus, Fortaleza, Ceará, Brazil.

Copyright $(2021$ Author(s) retain the copyright of this article. This article is published under the terms of the Creative Commons Attribution Liscense 4.0.
} 


\section{Material and methods}

\subsection{Approaches}

Medical examinations were recognized using a mixture of adherent terms and MeSH terms: cerebellar ataxia, ataxia, physical rehabilitation, physical treatment, training, exercise, rehabilitation, allied well-being treatment, speech treatment, language treatment, voice treatment, as well as work treatment. The duration of the time chosen was from January 1, 1980 to December 31, 2011, and the articles had to be published in English or Dutch.

\subsection{Choice}

The articles consisted of potential medical tests that reviewed the efficacy of an allied treatment of health and wellbeing (i.e., physical treatment, speech and language treatment or work treatment) in individuals with cerebellar ataxia, without conflicting comorbidity. Research that analyzed individuals with and without cerebellar ataxia consisted only of whether private information for people with cerebellar ataxia can be extracted.

\subsection{Proof classification}

Both the superior quality of the research layout and the verdicts were evaluated according to the category of the degrees of proof using the EBRO category of the Dutch Cochrane Center, as well as the Dutch Institute for Health Care Renewal (CBO), a participant in the Standards International Network (GIN).

Due to considerable diversification among the included research studies involving a concern with the severity of the disease, therapy objectives, treatments, duration of follow-up and results measures, and a meta-analysis may not be done.

\section{Results and discussion}

Up to 409 recommendations per data source search were discovered, of which 33 articles were selected. In highlight, 5 articles were determined with reference checklists. Of the 26 studies, 3 did not involve training, but focused only on a simply quantifiable research with kinematic impact of solitary treatments, and were omitted because of this [4-6]. Of the 13 status records chosen [7-19], only 7 can be combined as a result of comparable therapy techniques [9,14,18-22]. The studies included were heterogeneous concerning diagnosis, treatments, as well as outcomes. The treatments focused on numerous cerebellar deficiencies and limitations in everyday life, as well as differing in regularity, period and types of exercises.

\subsection{Physical treatment}

Fifteen research studies examined the impacts of physical treatment [8,11-15, 20, 22-31], of which [20,23-22,25-28,30], physical treatment commonly focuses on up to more than one domain name, for example, step, balance, control, position, and muscle strengthening. The treatments used were composed of disadvantageous physical treatment sessions, computer-aided training, treadmill training and psychophysiological feedback treatment.

\subsection{Standard physical treatment exercises}

Traditional physical treatment aims at least between the domain names in the list below: balance, stride, synchronization, resistance as well as position. Most physical treatments and help (e.g., a walking stick or a walking structure) and physical treatment devices (e.g., a balance round, weights or a treadmill) were used.

In a research study integrating physical treatment with work-related treatment in 42 clients with degenerative cerebellar ataxia, physical treatment, with an emphasis on balance, gait, basic problem, muscle tissue resistance and movement disposition, led to a decrease in the severity of ataxia as well as fall regularity, as well as an increase in the rate of gait and tasks of everyday life (QVT), in contrast to $50 \%$ of people $(n=21)$ who obtained the same therapy 4 weeks later. The improvement was more popular in trunk ataxia than in arm or leg ataxia. Another research study in individuals with degenerative cerebellar ataxia, consisting of 10 individuals with primary brain outcomes, as well as 6 clients with primary sensory ataxia.

Considerable renewals in QVT and gait and balance criteria, as well as a decrease in ataxia extension were observed after 4 weeks of training, compared to the standard. The impacts of training were much more distinct for clients with intact sensory course methods [26-27]. 
A research study was conducted in 26 clients with ataxia due to multiple sclerosis (MS), mixed control exercises, gait training, balance training and vestibular exercises. Arm or leg ataxia was more immune to physical treatment than trunk ataxia.

A survey of 37 MS patients with ataxia found that physical treatment with weighing, along with occupational treatment, caused renewal of practical capacity, worrying QVTs, tiredness, as well as physical function, in contrast to people $(\mathrm{n}=$ 9) who did not receive any type of treatment. The physical treatment was concentrated in the advertising of typical pose, as well as movement, using weight support, cushioning and weighting, joint estimation and compression, and boosting automated equilibrium reactions using a gymnastic sphere [28].

\subsection{Treadmill training}

Locomotor training with the aid of body weight on a treadmill and walking on the ground in a person with traumatic mental injury leads to improved balance, gait specifications, electric motor work, as well as isometric resistance examinations of the trunk. In a young person with severe ataxia after a cerebellar infarction, body weight assistance on a treadmill, as well as during land walks, increased walking and transfer abilities [8].

\subsection{Relaxation and treatment of psychophysiological feedback}

Three short articles recommended a favorable impact of psychophysiological feedback treatment in individuals with ataxia. For example, collection using electromyogram (EMG) psychophysiological feedback in 3 people with ataxia due to numerous sclerosis and degenerative cerebellar ataxia, the topics were able to minimize ataxia [14-15,24].

\subsection{Computer-aided training}

Improved control of the upper arm or legs after training with flexible robotic treatment was recommended in a research study of 8 patients with multiple sclerosis with upper arm or leg ataxia. The training contained the execution of flat actions, while performing a treatment of a robot, which produced pressures that decreased or improved the curvature of activities [22].

\subsection{Supervising sports activities}

One survey found that customers came from the rate ratio on arm or leg aiming activities, balance and manual dexterity after climbing training. The 4 individuals had upper and reduced arm or leg ataxia for various reasons obtained [29].

\subsection{Work treatment}

Hamilton's ratings for clinical depression increased, but disability ratings, as well as quality of life ratings, remained safe $[22,31-36,28,30]$.

The work treatment, incorporated into the physical treatment, consisting of the stipulation of tools and recommendations on language development evaluation (LDE) works caused a considerable renewal in both the rate and the ability to complete LDE work in a survey with 37 clients with ataxia due to numerous sclerosis [28].

Extensive recovery with work-related treatment combined with physical treatment proved advantageous in a study of 42 individuals with degenerative cerebellar ataxia. The work treatment focused on QVT, relaxation, individual health, but similarly in balance exercises, coordinated the work of the arm or legs and trunk, and those of double electric motors.

\section{Conclusion}

In a Spanish survey on degenerative cerebellar ataxias, health and wellness treatment expenses were estimated at about $\$ 24,500$ per person per year. Although the result of such treatments is prepared, and currently experienced as such by health care staff as well as clients in everyday technique, there is a lack of high-quality professional tests of the results of allied health and wellness treatments in clients with cerebellar ataxia.

Of all the allied self-control, the effectiveness of physical treatment was actually more evaluated. Given that balance problems are a feature of ataxia, it has been anticipated that, for those self-controls, the greatest proof would certainly be discovered. Based on the reasonably low quality of research that has passed on our addition requirements, we have re-examined the retrospective research we have actually left out. 
Basically, the treatment was customized for the person, who is in tune with the concepts of healing, but hinders generalizing these therapeutic procedures. We actually accept here the different etiologies of cerebellar ataxias from each other, but we value that facets such as comorbid function or innate ability to improve certainly vary according to condition. For future research, it may be helpful to take a look at whether the visibility of depressive signs and symptoms affects the efficiency of such treatments, or whether viable functional renewals are driven by a decreased impact of cognitive disorders, which can coexist in various diseases underlying ataxia, needs to be discovered, as all studies consulted consisted of research reporting a favorable outcome.

What are the helpful suggestions? Physical treatment, as well as work-related treatment, is suggested in the cerebellar condition, based on grade 2 verdicts, due to the fact of the selection of typically personalized treatments, as well as because many of the treatments have not been explained in complete information, being difficult, at this stage, to provide a detailed standard for such a physical treatment program. Reasonably better research recommends that the program needs to be extensive and its requirements, changed, as well as the individual's restrictions, that patients should be encouraged to exercise at home and that therapy should begin in the earlier stages of the condition because those individuals seem to be much more likely to evolve the clinical picture. Much better is to promote the development of the drug area, as well as to perform randomized and regulated tests that discover the impact of allied wellness treatments on neurological problems such as cerebellar ataxia.

\section{Compliance with ethical standards}

\section{Disclosure of conflict of interest}

The authors declare that there is no conflict of interest.

\section{Statement of ethical approval}

This type of study does not need ethical approval.

\section{References}

[1] Lacourse MG, Turner JA, Randolph-Orr E, Schandler SL, CohenMJ. Cerebral and cerebellar sensorimotor plasticity fol- lowing motor imagery-based mental practice of a sequential movement. J Rehabil Res Dev. 2004; 41: 50524.

[2] Martin TA, Keating JG, Goodkin HP, Bastian AJ, Thach WT. Throwing while looking through prisms. I. Focal olivo cerebellar lesions impair adaptation. Brain. 1996; 119(4): 1183-98.

[3] Matsumura M, Sadato N, Kochiyama T, Nakamura S, Naito E, Matsunami K, Kawashima R, Fukuda H, Yonekura Y.Role of the cerebellum in implicit motor skill learning: a PET study. Brain Res Bull. 2004; 63: 471-83.

[4] Baram Y, Miller A. Virtual reality cues for improvementof gait in patients with multiple sclerosis. Neurology. 2006; 66: 178-81.

[5] Clopton N, Schultz D, Boren C, Porter J, Brillhart T.Effects of axial weight loading on gait for subjects with cerebellarataxia: preliminary findings. Neurol Rep. 2003; 27: 15-21.

[6] Okajima Y, Chino N, Noda Y, Takahashi H. Accelero- metric evaluation of ataxic gait: therapeutic uses of weighting andelastic bandage. Int Disabil Stud. 1990; 12: 165-8.

[7] Ada L, Sherrington C, Canning CG, Dean CM, Scianni A. Computerized tracking to train dexterity after cerebellar tumour: a single-case experimental study. Brain Inj. 2009; 23: 702-6.

[8] Cernak K, Stevens V, Price R, Shumway-Cook A. Loco- motor training using body-weight support on a treadmill in conjunction with ongoing physical therapy in a child with severe cerebellar ataxia. Phys Ther. 2008; 88: 8897.

[9] Dordel HJ. Mobility training following brain trauma- results of intensive individual treatment. Int J Rehabil Res. 1987; 10: 279-90.

[10] Fillyaw MJ, Ades PA. Endurance exercise training in Friedreich ataxia. Arch Phys Med Rehabil. 1989; 70: 78688.

[11] Freund JE, Stetts DM. Use of trunk stabilization and locomotor training in an adult with cerebellar ataxia: a single system design. Physiother Theory Pract. 2010; 26: 447-58. 
[12] Gibson-Horn C. Balance-based torso-weighting in a patient with ataxia and multiple sclerosis: a case report. J Neurol Phys Ther. 2008; 32: 139-46.

[13] Goulipian C, Bensoussan L, Viton JM, Milhe-De Bovis V, Ramon J, Delarque A. Orthopaedic shoes improve gait in Friedreich's ataxia: a clinical and quantified case study. Eur J Phys Rehabil Med. 2008; 44: 93-8.

[14] Guercio J, Chittum R, McMorrow M. Self-management in the treatment of ataxia: a case study in reducing ataxic tremor through relaxation and biofeedback. Brain Inj. 1997; 11: 353-62.

[15] Guercio JM, Ferguson KE, McMorrow MJ (2001) Increasingfunctional communication through relaxation training and neu- romuscular feedback. Brain Inj. 2001; 15: 1073-82.

[16] Harris-Love MO, Siegel KL, Paul SM, Benson K. Reha- bilitation management of Friedreich ataxia: lower extremity force-control variability and gait performance. Neurorehabil Neural Repair. 2004; 18: 117-24.

[17] Landers M, Adams M, Acosta K, Fox A. Challenge-ori- ented gait and balance training in sporadic olivopontocerebellar atrophy: a case study. J Neurol Phys Ther. 2009; 33: 160-8.

[18] Perlmutter E, Gregory PC. Rehabilitation treatment options for a patient with paraneoplastic cerebellar degeneration. Am J Phys Med Rehabil? 2003; 82: 158-62.

[19] Sapir S, Spielman J, Ramig LO, Hinds SL, Countryman S, Fox C,Story B. Effects of intensive voice treatment (the LeeSilverman Voice Treatment [LSVT]) on ataxic dysarthria: a case study. Am J Speech Lang Pathol. 2003; 12: 387-99.

[20] Armutlu K, Karabudak R, Nurlu G. Physiotherapy approaches in the treatment of ataxic multiple sclerosis: a pilot study. Neurorehabil Neural Repair. 2001; 15: 203-11.

[21] Mulligan H, Mills K, Pascoe 0, Smith M. Physiotherapy treatment for a child with non-progressive congenital ataxia. NewZealand J Physiother. 1999; 27(3): 34-41.

[22] Vergaro E, Squeri V, Brichetto G, Casadio M, Morasso P, Solaro C, Sanguineti V. Adaptive robot training for the treatment of incoordination in Multiple Sclerosis. J Neuroeng Rehabil. 2010; 7: 37.

[23] Balliet R, Harbst KB, Kim D, Stewart RV. Retraining of functional gait through the reduction of upper extremity weight- bearing in chronic cerebellar ataxia. Int Rehabil Med. 1987; 8: 148-53.

[24] Davis AE, Lee RG. EMG biofeedback in patients with motor disorders: an aid for co-ordinating activity in antagonistic muscle groups. Can J Neurol Sci. 1980; 7: 199-206.

[25] Folz T, Sinaki M. A Nouveau aid for posture training in degenerative disorders of the central nervous system. J Musculoskelet Pain. 1995; 3(4): 59-69.

[26] Ilg W, Brotz D, Burkard S, Giese MA, Schols L, Synofzik M. Long-term effects of coordinative training in degenerativecerebellar disease. Mov Disord. 2010; 25: 2239-46.

[27] Ilg W, Synofzik M, Brotz D, Burkard S, Giese MA, Schols L. Intensive coordinative training improves motor performance in degenerative cerebellar disease. Neurology. 2009; 73: 1823-30.

[28] Jones L, Lewis Y, Harrison J, Wiles C. The effectiveness of occupational therapy and physiotherapy in multiple sclerosis patients with ataxia of the upper limb and trunk. Clin Rehabil. 1996; 10: 277-82.

[29] Stephan MA, Krattinger S, Pasquier J, Bashir S, Fournier T,Ruegg DG, Diserens K. Effect of long-term climbing training on cerebellar ataxia: a case series. Rehabil Res Pract. 2011; 525879.

[30] Miyai I, Ito M, Hattori N, Mihara M, Hatakenaka M, Yagura H, Sobue G, Nishizawa M. Cerebellar ataxia rehabilitation trial in degenerative cerebellar diseases. Neurorehabil Neural Repair. 2011; 26(5): 515-22.

[31] Vaz DV, Schettino Rde C, Rolla de Castro TR, Teixeira VR, Cavalcanti Furtado SR, de Mello Figueiredo E. Treadmill training for ataxic patients: a single-subject experimental design. Clin Rehabil. 2008; 22: 234-41.

[32] Silva RC, Saute JA, Silva AC, Coutinho AC, Saraiva-Pereira ML,Jardim LB. Occupational therapy in spinocerebellar ataxia type 3: an open-label trial. Braz J Med Biol Res. 2010; 43: 537-42.

[33] Sliwa JA, Thatcher S, Jet J. Paraneoplastic subacute cer- ebellar degeneration: functional improvement and the role ofrehabilitation. Arch Phys Med Rehabil. 1994; 75: 355-7.

[34] Smith MA, Shadmehr R. Intact ability to learn internal models of arm dynamics in Huntington's disease but not cere- bellar degeneration. J Neurophysiol. 2005; 93: 2809-21. 
[35] Stoykov ME, Stojakovich M, Stevens JÁ. Beneficial effects of postural intervention on prehensile action for an individual with ataxia resulting from brainstem stroke. Neuro Rehabilitation. 2005; 20: 85-9.

[36] Werner S, Bock O. Mechanisms for visuomotor adaptationto left-right reversed vision. Hum Mov Sci. 2010; 29: $172-8$. 